\title{
Tumores y quistes del ovario en las niñas
}

Los tumores y quistes del ovario (T. Q. O.) constituyen una patología poco frecuente en Ias niñas (I). En los grandes Hospitales de niños en EE. eU. se describen de 1 a 4 casos por año. En revisiones anteriores de autores nacionales $(2,3,10)$, se constata este mismo hecho. Cuando esta patología se presenta en los servicios de cirugía pediatrica o en los servicios de urgencia, crea con frecuencia problemas de diagnóstico y tratamiento, lo que nos ha motivado para revisar la experiencia del Servicio de Cirugía Pediátrica del Hospital Roberto del Rfo en los viltimos 13 años.

\section{MATERIAL Y METODO}

Se revisaron las historias clínicas de las enfermas que egresaron entre los años 1962 y 1975, con el diagnóstico de T. o Q. O., que tentan confirmación clínica en la intervención quirúrgica y clasificación histopatológica. Se encontraron 22 casos: 15 tumores y 7 quistes.

\section{RESULTADOS}

Para comprender mejor los resultados, hemos analizado tas clasificaciones de los T. $\mathbf{Q}$. $\mathbf{O}$. adoptando un resumen de la clasificación práctica tomada de Novak-Jones (4)

\footnotetext{
- Servicio de Cirugía Infautil, Hosp. Roberto del Rfo. - -Servicio de Anatomfa Patológica, Hosp. Roberto del Rlo.
}

Rev. Chilena de Pediatría, Vol. 47, No 1, 1976
Drs. Julio Aguirre Fuentes*, Femando Torres Kay", Jaime Cerda Sepulveda" y David Mirkin Wexman*"

\section{CLASIFICACION}

\section{A. TUMORES BENIGNOS}

\section{Quisticos}

a) No Neoplásicos (Q. folicular-Q. luteínico)

b) Neoplásicos (Cistoadenomas)

2. Sólidos (Fibroma-Linfangioma.Osteo. condroma).

\section{B. TUMORES MALIGNOS}

1. Quisticos (Cisto Adeno Carcinoma)

2. Sólidos (Carcinoma-Teratoma-SarcomaLinfoma)

\section{Con polencial endoctino}

a) Poco Malignos (Disgerminoma-T. Cel. granulosas, Arrenoblastoma)

b) Muy Malignos (Corion epiteliona)

\section{Metastásicos}

En Ia muestra examinada se encontraron I5 casos de $T$. $O$; d de estos, 12 eran teratomas benignos y 3 tumores malignos, 1 tumor de células granulosas y I linfoma. (Tabla l) .

Quiste ovárico se encontró en 7 enfermas, de los cuales 6 eran $Q$. foliculares y I $Q$. seroso simple (Probablemente también folicular). (Tabla 1). 


\section{A. TUMORFS (l’́ casos)}

\section{Teratomas ( 12 casos)}

Son tumores sóliclos, con cavidades quísticas, de tamaño pequeño o mediano derivados de células germinales y se caracterizan por estar formados por estrucuras procedentes de las 3 capas emtrionarias. (4) En nucstros casos todos tenian picl, pelos, hueso o sartílagro, tejido nervioso, músculo o tejitlo respinatorio.

Tarial

TUMORES Y QUISTES THET, OVARIO (Hosp. R. del Rio, J962-1975)

\begin{tabular}{lc}
\hline Diagnostico & $\begin{array}{c}\text { Námero ile } \\
\text { rasos }\end{array}$ \\
\hline Tumorcs lenignos & 12 \\
Tumores malignos & 3 \\
Quistes & 7 \\
\hline Total & ga2 \\
\hline
\end{tabular}

A pesar de gue los Teratomas se clasifican dentro de los Tumores Malignos (5), ch ntes. tros 12 casos no se encontró en c1 estulio histo-patológico, signos de maliznidlid.

\section{Tumores matignos}

a) Disgerminoma (l cass) Es un tumop sóliclo de pequeño tamaño, (ampue puele at. canzar grandes proportions) derivalo ale céIulas germinativas de temprano desarrollo enbrionario, no tiene en general activistad chtocrina y su malignirlad es baj.t. Ciura latbiualmente con el trataniento quirúgice conservador, el sue da ma supervivencia de 80 a $90 \%$ a los 5 años (8).

b) Tumor de colulas granulosas (1 caso) Es un tumor sólido de pequeño, mediamo o gran tamaño, de superficic lisa o abollonada, en general bien capsulado, de color gris amarillento y por ser derivado del estroma gonadal, tiene carácter funcionante (feminizante) (6).

c) Linfoma (1 caso) Tumor de muy escasa frecuencia entre los T. O.(7) y de gran malignidad, en nuestro caso se trataba dé un tumor abollonado que invadía los órganos vecinos.
B. OYYSTES (f casos)

I.os quistes Foliculares son estructuris quísticas no neoplásicas y funcionales que se desarrollan a partir de Foliculos atrésicos. Su ta. maño habialalmente es moderaclo y su evolución normal es a la reabiorción espontínea. lin algunos casos, sin cmbargo, pueden complicarse y dar sintomalogia aguda riolenta (1).

Distribucion por colates (Figura No 1).

In nuestra serie los T. (2. O. se cocontraban en todas las exlatc's, descle 1 R. N. de 17 días lassi: los 1 t años.

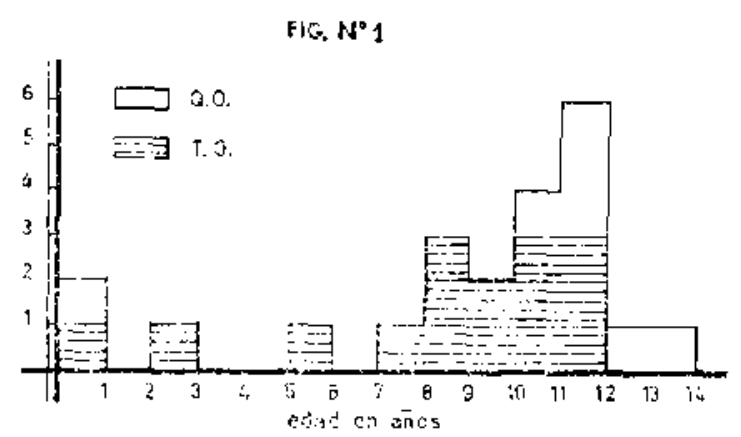

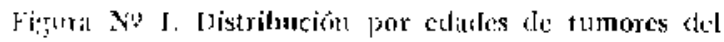
antio (T. O.) y quistes del orario (Q. O.)

Los T. O. se encontraron con mayor stecuchciat entre los 8 y 12 años y un $86 \%$ tení. más de 7 años. El caso de menot edad correspondió a un lactante de 9 meses, intervenislo de. ursichcia prot un Jeatoma torcido.

Los Q. O. se presentan con mayor frecusitsia entre los 10 y 11 años. El saso de um R. $N$. de 17 dias correspondia a un quisie seroso que provecó una obs'rucción intesti1131.

\section{Sintomatologia (Tablat 2).}

El cuadro clínico en general es simple y la historia gira en torno al clolor abclominal y la jresencia de una masa tumoral (9) .

\section{Dolor}

Fstuwo presente en 21 de los 22 casos $(94,5 \%)$ y se presentó en dos formas: a) dolor agudo, 
Nitncro di?

casos

\begin{tabular}{lc}
\hline Dolor Abdominal & 21, \\
I'urior Abclominal & 19 \\
Vómitos & 8 \\
Ficbre & 1 \\
Constipación & 1 \\
Disurin & 1 \\
\hline
\end{tabular}

intenso, de tipo cólico, localizado en el abdomen inferior y frecuentemente refericlos a la F. I. D. de corta evolución (hor.ss o días) y que no cede a analgésicos o int espasmótlicos. Este tiox de dolor cotá generalmente asmiat(lo : una complicación. b) (Iolor suave, moclerarto, intermitente o progresiyn de evolucion en dias o ne: $e_{3}$, Este dolor "virespond: al crecimicnto de la masa umoral o a li cont. presión de órganos vecinos.

\section{Mata Lmoral}

Se encontio en el $100 \%$ ale Jos Tumores y an cl $5 \pi, 1 \%$ Id los quistes.

La tumoración abdominal fus notada pos la enferma o fnmiliares, o comprobado al exitmen físico de ingreso. En un raso constiuyc un hallazgo de examen al consultar la confcrma por otra patología (amisclalitis ag. $\mathrm{cm}$ el Servicio de Lrgencia.

La misa abdominal cstát representada por ubicada en cl hipogasirio, gue a veces alcanza una tumoración de mediano o gran tamaĩo, hasta cl ombligo, de consistencia dura, dolorosa, redondeada, fija o desplazable y yuc generalmente se comprueba con un cuidadoso linco rectal.

\section{Vómitos}

Solo tl $40 \%$ de las enfermas presentaron vómitos y siempre estuvo acompañando a las crisis dolorosas.

\section{Ficbre-Diswia-Constipación}

Sólo se encontró en una enferma.
Sc describe en la enferma portadora de dis germinoma, tumor maligno que terminó con la vida de la paciente en corto periodo.

Dicignósico pre-operatorio (Talula 3)

El diagnóstico de T. o (2. O. se planter on I1 de ay enformas (69,60\%). Fin 6 cosos se hizo

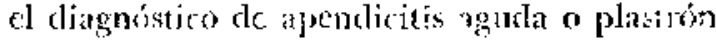

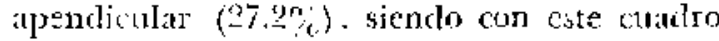
(c) el gue se piantea on milyol frecuencia el diagncytion diferencial. I as otres diaemostico be ingreso fuerom: amom abdominal (t casos), quiste abclominal (I), sarcona abciomina! (1)

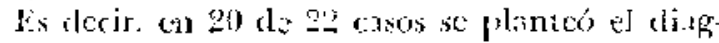
nóstico de thanor o quiste en el primer nom mento.

Tabla 3

\begin{tabular}{|c|c|}
\hline $\begin{array}{l}\text { Whatedistico } \\
\text { meateretoro }\end{array}$ & $\begin{array}{l}\text { No die } \\
\text { canos }\end{array}$ \\
\hline ? ?uiste ovárico & 14 \\
\hline Aponciucitis ay. & 6 \\
\hline 'I'umor abdominal & 4 \\
\hline Quistr: abdomindal & 1 \\
\hline Sarcoma alydominal & 1 \\
\hline Embariazo & l \\
\hline Hitleomedrat & 1 \\
\hline
\end{tabular}

Tirmpo de cablucion de los sintomas (Tabla 4)

Los sintomas de 2 enfermas ( 1 T.,$~ l ~ O)$ ) tonían menos de 12 looras de cyolurion al consultar. El major número de casos tenía sintomatología de varios díis de evolución

Tabli 4

\begin{tabular}{lcc}
\hline $\begin{array}{l}\text { Tiempo de evolución } \\
\text { de los sintomas }\end{array}$ & \multicolumn{2}{c}{ No de } \\
& \multicolumn{2}{c}{ casos } \\
& $T$ & $Q$ \\
\hline Hotas & & 1 \\
Dias & 1 & 1 \\
Meses & 6 & 6 \\
Ignorado & 6 & \\
\hline
\end{tabular}




\section{Operarión efectuada (Tabla 5)}

En 15 cle los 22 casos $(68,2 \% / 6)$, la intervención quirúrgica constituyó uná "urgencia", efectuándose la operación en las primeras 2t horas del ingrcso. De estos, 10 eran tumores y 5 quistes. Fn la intervención de estos 15 casos, en 11 ( $73 \%)$ se comprobó la existencia de una o más complicaciones.

\section{Tabla 5}

\begin{tabular}{|c|c|c|}
\hline \multirow[t]{2}{*}{ Operación efectuata } & \multicolumn{2}{|c|}{$\begin{array}{l}\text { No de } \\
\text { casos }\end{array}$} \\
\hline & 7 & $Q$ \\
\hline Salpingo-Ooforcctom ía & 13 & $\mathbf{l}$ \\
\hline Ooforcctonía & 2 & 4 \\
\hline Quistcetomía & & 1 \\
\hline Apendiccctomúa & 5 & 3 \\
\hline
\end{tabular}

\section{Infarofomias (Talbla lib)}

De clerción se usaron linjarocomias (ransversales, predominando la de Pranuentiel que da un excelente campo operatorio de la zona pelviana. I ats laparotomías tlansversas supra o infra unbilicales, usadas cn 15 de 22 casos. permitcs una amplia exposición y explora ción abdominat, detcrminando además, menor número de conplyicaciones de ricatrización.

Tabla 6

\begin{tabular}{lc}
\hline Iaparotomia & $\begin{array}{r}\text { No de } \\
\text { casos }\end{array}$ \\
\hline Pfannensticl & 8 \\
Transwcrsa & 7 \\
Mc. Burncy & 5 \\
Meclia I. V. & 1 \\
Oblicua izq. & 1 \\
\hline
\end{tabular}

En 5 aasos se usó incisión de Mc Burney y corresponde a en[ermas operadas con diagnóstico de Apendicitis aguda, en las que logró extirpar el quiste ampliando la incisión.

\section{Técnira operatovia}

El tratamients quirúrgico de los T. O. consistió en la salpingo-ooforectomía en 13 casos y
1. ooforectonlía en 2 casos. Estas técnicas cons. tituyen el tratamiento habitual en tste tipo de lesiones, criterio conservador, basado en el hecho de que estas lesiones, son poco invasoras y de poca capacidad metastasiante (11).

\section{Evolucion}

Los 12 teratomas benignos, en que se practicó salpingo-coforectomia en $10 \mathrm{y}$ ooforectomía en 2, han sido controlados en general por muy poco tiempo, que no va más allá de 9 mesês, perclienclose la mayoría clespués de 1 mes de control.

De los 3 T. O. malignos operados, en que se practicó salpingo-ooforestomía el único bajo control es la enferma de lumor de células granulosas operada en febrero de 1975 y que a $\operatorname{los} 9$ mexes de evolución se encuentra en buenas condiciones.

Una enferma portaclora de un disgerminoma del ovario, operada en mar7o de 1968, en quién el tumor pesaba $1.750 \mathrm{gr}$. y se practicó salpingo-ooforectomia, recidivó localmente a los 3 meses, presentando posteriormente metástasis rerebrales que la llevaton rápidamente a la muerte.

La enferna que presentó un linfoma del ovario, se perdió de control después de ser enviada en interconsulta âl Instituto de cl Radium.

En las enfermas portadoras de quistes foliculares, el tratamiento consistió en la ooforectomít en 4 casos y salpingo-ooforectomía $\mathrm{En}$ 1 caso, ambas lérnicas no adecuadas a la naturaleza cle la lesión, ya que lo indicado en estos casos debió ser la Quistectomia, el drenaje del quiste, la resección en cuña o la marsupialización, tratando de conservar al máximo el tejido ovárico sano.

\section{Complicaciones}

Se encontro complicaciones en 15 de 22 casos $(68,2 \%)$ siendo la torsión la más frecuente. De los 15 T.O. 7 estaban torciclos, y 1 de estos además estabá roto y proclujo hemoperitoneo de más de $500 \mathrm{c}$., ingresando la enferma con un cuadro de anemia aguda. 
No se encontró quistes ováricos torcidos.

En 5 casos de Q.O. se comprobó rotura del quuiste, estando I de estos casos acompañado rie hemoperitoneo moderado.

Un .Q.O. operado en un R.N. de 17 dias de edad, pesaba 250 gr. y produjo un cuadro de Ostrucción Intestinal que motivó una intersención quirúrgica de urgencia.

\section{RESUMEN}

En el periodo comprendiclo entre 1962 y 1975 , se han intervenido en el Servicio de Cirugía del Hospital de Niños Roberto del Río, 22 casos de tumores y quistes ováricos. De éstos 15 son tumores, 12 teratomas benignos y 3 tumores malignos, (1 clisgerminoma, I cumor de células granulosas y 1 linfoma).

Quiste ovárico se encontró en 7 casos.

Se analizan la distribución por edacles, el cuadro clínico, Ja oportunidad y certeza clel diagnóstico, el tratamien o efcctuado y las complicaciones.

Fallecidos 2: 1 linfoma y I clisgerminoma.

\section{SUMMARY}

The expericnce about Oyarian Cyst an Tiymots, within the years 1962-1975, in the $\dot{P}_{\epsilon}$ diatric: Surgery Unit at the Roberto del Rio Children's Hospital, is analized.

In the last thirteen years 22 girls were operated with the above clinical diagnosis, that was afterwarls confirmated in all the cases by the pathologyst. AlI the patient were under I4 years old, and most of them between 8 and 12 years old. The youngest was 17 days old.

The clinical picture was characterized by a great mass in the lower abdomen, and intence colic pain located in the same area, specia$\mathrm{lly}$ in the right iliac fosa.

This symptomatologic induced to an emergency operation in 15 patients, the preoperative diagnosis was most of the cases correct, with the only exception of confussion with acute appendicitis. Most of the tumors were complicated by torsion and subcquient hemowhage and hemoperitoneum.

The postoperative study of the 22 cases, slowed 15 tumors and 7 ovarian cyst of the tumors 12 were bening teratomas, and $5 \mathrm{ma}$ lingnant tumors (1 dysgerminoma, 1 limphoma, and I granuloma cell tumors).

Two girls died, both with malignant tumors (dysgerminoma and limploma).

The surgical procedure consists in laparatomy with resection of the cyst or tumors, and ooforectumia in same cases.

\section{REFERENCIAS}

1. Towne B. et al. Oyarian Cysts and Tumors in Infancy and Childhood. J. Periatr. Surg. 10: 311 , 1975.

2. Ariztía R. y A. Gantz. Tumores de Ovario en la Infancia. Tesis 1949. Arch. Hosp, Roberto del Río. p. กी, 1952.

3. Espinora J. y R. Artiga. Tutnores del Ovario en la Infancia. Rev, Chíl. Pediatr. 27: 454, 1956.

4. Novak-Joncs. Ginecología. 8n edición, Baltimore. Fditorial Interamericana, 1971, p. 448.

5. Williams I. G. Tunores en el Niño. Bancelona. Eslit. JIMS, 1978.

6. Ein S. H. Malignant ovarian tumors in children. J. Pediatr. Sturg. 8: 539, 1979.

7. Pochedy C. and T. Necheles. Major Problems in Childhood. Springfield. Ch. C. Thomas Publisher, $19 \% 3$.

9. Sutow W, et al. Clinical Pediatric Oncology. Saint Iouis, C. V. Mosby Company, 1973.

9. Bucket J. ct al. Orarian Tumars. Seminars OncoIng? 1: 74, 1974.

10. Villagrăn G. Quiste Ovárico en un Neonato. Rev. Chil. Pediatr. 14: 199, 1971.

11. Harris H. Rational surgery for tumor of the ovary in children. J. Pediatr. Surg. 9: 289, 1974 